(4) dead eggs each containing a single dead parasite and dead eggs containing a live parasite, she usually deposits in the former.

It should be stated that the female Schedius deposits her egg in the host with a portion of the pedicel protruding through the gipsy-moth egg-shell, this probably performing a respiratory function. To eliminate this as a means of distinction between parasitised and unparasitised eggs, all pedicels were removed with a sharp scalpel before the experiments. These preliminary experiments suggest that the oviposition response in Schedius cannot be due to any simple stimulus. It is hoped to investigate the conditions under which this selective faculty is impaired or destroyed.

Farnham House Laboratory,
Farnham Royal,

D. C. Lroyd.

Slough, Bucks.

Feb. 7.

${ }^{1}$ Salt, G., Proc. Roy. Soc., B, 114, 455; 1934.

\section{Diet of Seals}

THe recent illegal slaughter of grey seals in the supposed interests of the Cornish fishermen directs attention to the diet of seals. On what do they usually feed? On fish, as the fishermen and their friends assert, or on some of the other creatures which abound in the waters of the ocean?

Obviously the diet of seals, like that of whales, can be ascertained only by opening their stomachs and examining their contents. In the case of the arctic and antarctic seals this has been done to a considerable extent.

Nansen $^{1}$, R. Brown ${ }^{2}$, Kumlein ${ }^{3}$, Malmgren ${ }^{4}$ and Chapman Spencer ${ }^{5}$ all testify to the extent to which the arctic seals feed on ereatures other than fish-squid, crustaceans and shellfish-and, in the case of the antarctic seals, the evidence of E. A. Wilson ${ }^{6}$ and Matthews ${ }^{7}$ is equally strong.

As to the diet of the grey seal, there seems to be very little scientific evidence beyond that which is contained in Mr. Steven's report ${ }^{8}$. Unfortunately, Mr. Steven was only able to examine the stomach contents of three seals; there were fish remains in two, and a cuttle-fish beak in a third. The examination by him of a greater number of stomachs might have led to the discovery of more remains other than those of fish, that is, those of crustaceans and shellfish, and might have still further weakened the case against the grey seal.

Until the matter is more fully investigated, the destruction of grey seals on the plea that they destroy large quantities of food-fish does not seem to be justified.

\section{Exmouth.}

R. W. GRAY.

Feb. 19.

1. Nansen, "Hunting and Adventure in the Arctic", p. 267.

"Brown, R., "On the Seals of Greenland", Proc. Żool. Soc., Lond., p. $411 ; 1868$.

Kumlein, L., "Report of the Howgate Polar Expedition".

- Malmgren, Arch. Naturg., p. 75; 1864.

5 Chapman Spencer, "Watkins's last Expedition", pp. 116, 134, 223 and 280 .

"Wilson, E. A., "National Antaretic Expedition", 11, 13, 28, 36, 44 and 45 .

'Matthews' "Discovery" Report on the Natural History of the Elephant Seal.

Steven, G. A., "A Short Investigation into the Habits, Abundance, 作 A880c., 19, No. 2, 489-502; May 1934.

\section{Red 'Water-Bloom' in British Columbia Waters}

IN NATuRE of September 22, 1934, there is a communication from Mr. T. John Hart describing the occurrence of a red 'water-bloom' caused by Mesodinium in South African seas. It may be of interest to record an occurrence of blood-red water at Nanaimo, British Columbia, during the week of April 28, 1933. The water in a channel immediately north of the harbour was coloured crimson red in great patches. Examination of a sample of the water revealed a pure culture of a ciliate, identified by $\mathrm{Mr}$. G. H. Wailes as Mesodinium rubrum, Lohmann.

About this time oysters in Ladysmith Harbour, fifteen miles south of Nanaimo, were reported to contain red 'worms'. Investigation disclosed the fact that the crystalline styles were coated with a red colouring matter, evidently as a result of feeding upon Mesodinium. Examination of the styles of local clams showed a similar condition.

The appearance of this 'bloom' of Mesodinium followed a period of a couple of weeks of bright, sunny, calm weather. No discoloration was observed in 1934 .

Pacific Biological Station,

W. A. Clemens.

Nanaimo, B.C.

Jan. 31.

\section{Magnetic Measurement of Ionic Deformations in Crystals}

DURING the past few years, it has been shown that the diamagnetic susceptibilities of ions in crystals are approximately additive, and their values are in fairly close agreement with values calculated theoretically from the wave-mechanical structures of free ions ${ }^{1}$. Attempts to interpret the experimental results in more detail have been handicapped by a lack of essential data, for although results are now available for many substances, some have been obtained for solutions and others for crystals, and the two are not directly comparable. With the view of obtaining more precise information for a particular group of crystals, we have studied the susceptibilities of the alkaline halides, using a method of measurement previously described ${ }^{2}$.

We have been specially interested to see how closely the additivity principle holds in this group of crystals, and we find that in general it holds to within 1 per cent. The results for the rubidium halides are typical.

\begin{tabular}{lccc} 
Salt & \multicolumn{3}{c}{ Diamagnetic Susceptibilities $\chi \times 10^{6}$} \\
& (1) Obs. & (2) Calc. & $\Delta \chi,(1)-(2)$ \\
$\mathrm{RbF}$ & $31 \cdot 9$ & $31 \cdot 4$ & $-0 \cdot 5$ \\
$\mathrm{RbCl}$ & $46 \cdot 4$ & $46 \cdot 2$ & $-0 \cdot 2$ \\
$\mathrm{RbBr}$ & $56 \cdot 4$ & $56 \cdot 5$ & $0 \cdot 1$ \\
$\mathrm{RbI}$ & $72 \cdot 2$ & $72 \cdot 6$ & $0 \cdot 4$
\end{tabular}

The only important exceptions to the additivity rule are the chlorides, bromides and iodides of lithium and cæsium, for which the calculated values exceed the observed values by $1 \cdot 6,1 \cdot 5$ and $1 \cdot 3$ in the case of the lithium salts, and by $2 \cdot 7,2 \cdot 4$ and $3 \cdot 1$ in the case of the cæsium salts. For the other halides upon which measurements have been made in the course of this work, the differences are of the order of $0 \cdot 2-0 \cdot 3$.

Exceptional results might have been expected for the cæsium halides, since these crystals have a different structure from the other halides, all of 